\title{
Fijación percutánea con tornillos en fracturas de pelvis y acetábulo. Experiencia en el Hospital de Traumatología «Dr. Victorio de la Fuente Narváez»
}

\author{
Percutaneous screws fixation in pelvic and acetabular fractures. Experience \\ in the Traumatology Hospital «Dr. Victorio de la Fuente Narváez» \\ Leonel Nieto Lucio, ${ }^{*, \ddagger}$ Sindy Camacho González, ${ }^{*}$, Zamir Alejandro Zárate Ayup \\ ${ }^{*}$ Médico Especialista en Traumatología y Ortopedia; ${ }^{\ddagger}$ Jefe del Servicio de Cadera, Pelvis y Acetábulo; $\$$ Médico adscrito al Servicio \\ de Cadera, Pelvis y Acetábulo; "Médico en Adiestramiento del Servicio de Cadera, Pelvis y Acetábulo. \\ UMAE Hospital de Traumatología "Dr. Victorio de la Fuente Narváez", IMSS, Ciudad de México
}

\begin{abstract}
Resumen
Introducción: La fijación percutánea ofrece ventajas en relación con la reducción abierta en fracturas de pelvis y acetábulo. El objetivo de este artículo es describir la seguridad de la cirugía percutánea con tornillos en pacientes con fracturas de pelvis y acetábulo. Material y métodos: Se realizó un estudio observacional, retrospectivo, longitudinal y descriptivo. Se revisó el expediente de adultos hospitalizados con fracturas de pelvis y acetábulo con desplazamiento menor a $3 \mathrm{~mm}$, a quienes se les practicó una intervención quirúrgica percutánea del 2009 al 2018. Se estudió el mecanismo de lesión, traumatismos asociados, tipo de fractura, días de estancia en el nosocomio, muerte intrahospitalaria, complicaciones transquirúrgicas o postquirúrgicas y grado de consolidación ósea. Resultados: Se registraron 531 pacientes, 454 cumplieron con los criterios de inclusión. El promedio de edad fue de 41.7 años, el mecanismo de lesión de alta energía tuvo una mayor prevalencia, $52 \%$ de los analizados tuvieron alguna lesión asociada, la más frecuente en miembros pélvicos. La fractura más común fue la fractura tipo AO 61B2 con 38.1\%. Se tuvo un promedio de 10.7 días de estadía intrahospitalaria. Tuvieron alguna complicación 15 pacientes (3.3\%), siendo la principal la malposición del material de osteosíntesis. Dos pacientes presentaron muerte intrahospitalaria a causa de lesiones asociadas. De los pacientes, 100\% presentaron consolidación ósea grados III o IV en la clasificación de Montoya. Conclusiones: La cirugía percutánea en fracturas de pelvis y acetábulo es un procedimiento quirúrgico seguro, ya que se asocia con una baja mortalidad intrahospitalaria, tiene un bajo porcentaje de tropiezos, disminuye la estadía en el nosocomio y tiene una alta tasa de consolidación ósea. Es un método que requiere de entrenamientos especiales y tiene una curva de aprendizaje prolongada.
\end{abstract}

Palabras clave: Fractura de pelvis, fractura de acetábulo, fijación percutánea, tornillos, seguridad.

Nivel de evidencia: IV

\section{Abstract}

Introduction: Percutaneous fixation offers advantages in relation to the open reduction in pelvic and acetabulum fractures. The objective of this study is to describe the safety of percutaneous screw surgery in patients with pelvic and acetabulum fractures. Material and methods: An observational, retrospective, longitudinal and descriptive study was carried out. The clinical file of adult patients with pelvic and acetabulum fractures with a minor displacement of $3 \mathrm{~mm}$ was reviewed, they underwent percutaneous surgery from 2009 to 2018. The mechanism of injury, associated injuries, type of fracture, days of hospital stay, hospital death, trans- or postsurgical complications and bone consolidation were studied. Results: 531 patients were registered, 454 met the inclusion criteria. The average age was 41.7 years, high energy injury had the higher prevalence, $52 \%$ of the patients owns an associated lesion, the most frequent in pelvic limbs. The most common fracture was the AO 61B2 type with $38.1 \%$. There was an average of 10.7 days of hospital stay. Fifty patients (3.3\%) had some complications, the main one being the bad position of the screws. Two patients suffered hospital death due to associated

Correspondencia:

Leonel Nieto Lucio

E-mail: drleonelnieto@gmail.com

Recibido: 16-03-2021. Aceptado: 13-04-2021.
Citar como: Nieto LL, Camacho GS, Zárate AZA. Fijación percutánea con tornillos en fracturas de pelvis y acetábulo. Experiencia en el Hospital de Traumatología «Dr. Victorio de la Fuente Narváez». Orthotips. 2021; 17 (2): 82-89. https://dx.doi.org/10.35366/99901 
injuries. All patients presented bone consolidation grade III or IV of the Montoya classification. Conclusions: Percutaneous surgery in pelvic and acetabulum fractures is a safe surgical procedure since it is associated with low hospital mortality, has a small percentage of complications, decreases hospital stay and has a high rate of bone consolidation. It is a procedure that requires special training and has a long learning curve.

Keywords: Pelvic fracture, acetabulum fracture, percutaneous fixation, screws, security.

Level of evidence: IV

\section{Introducción}

Las fracturas del anillo pélvico representan aproximadamente $3 \%$ de todas las fracturas, con una incidencia de entre 20 y 35 casos por 100,000 habitantes, según diferentes investigaciones de base poblacional. La exigencia social actual impone una restauración perfecta en fracturas de pelvis y acetábulo, incluso después de un traumatismo grave. La restauración anatómica de las superficies articulares es el objetivo más importante.

La osteosíntesis percutánea de la pelvis y acetábulo engloba un conjunto de técnicas de sujeción ósea mediante abordajes mínimamente invasivos, introduciendo implantes pequeños, tornillos canulados, a través de incisiones de menos de $1 \mathrm{~cm}$. Dentro de la variedad de fijación percutánea es posible encontrar: tornillos iliosacros, tornillos en la columna anterior de acetábulo (anterógrado y retrogrado), en la columna posterior del acetábulo (tornillo ilioisquiático), tornillo mágico (magic screw) y tornillo a las ramas iliopúbicas (retrógrado) (Figura 1). ${ }^{1}$

El abordaje percutáneo se asocia con menos complicaciones que las técnicas abiertas, ya que ofrece un tiempo quirúrgico más corto, reduce los peligros relacionados con la exposición quirúrgica, disminuye la pérdida de sangre, existe menor lesión de los tejidos blandos y se asocia con disminución de la estancia intrahospitalaria. Los planos de los tejidos blandos permanecen sin dañarse, lo que posteriormente facilitaría la artroplastia total de cadera en caso de ser necesario. ${ }^{2,3}$ Sin embargo, la geometría acetabular hace que la inserción percutánea de tornillos sea un procedimiento difícil (Figuras 2 y 3). ${ }^{4}$

Las indicaciones para la fijación percutánea aún están en debate, pero incluirían:

- Fracturas sin desplazamiento o con desplazamiento menor a $3 \mathrm{~mm}$ o que se reducen con maniobras de reducción cerrada, pero que son potencialmente inestables. ${ }^{5}$

- Paciente politraumatizado, con daño importante de los tejidos blandos, fracturas expues- tas, que complicaría o evitaría las técnicas abiertas. . $^{5-7}$

- Se puede usar como complemento en la reducción abierta en patrones complejos de fracturas. ${ }^{6,7}$

Las complicaciones de la fijación pélvica percutánea son variadas y se dividen en errores relacionados con el cirujano, la técnica y el paciente. ${ }^{6}$ El tratamiento percutáneo de las lesiones del anillo pélvico inestable es un procedimiento técnicamente exigente con una curva de aprendizaje pronunciada (Figura 4). Una comprensión profunda de la anatomía pélvica, vascular y neurológica es vital para prevenir complicaciones graves. La colocación percutánea de los tornillos en fracturas de pelvis y acetábulo puede presentar complicaciones como el mal posicionamiento de los tornillos, mala reducción de la fractura, infección, riesgo de daño neurológico, vascular y estabilidad insuficiente, lo que puede provocar no unión o desanclaje del material de osteosíntesis y asociarse con cirugías de revisión. ${ }^{8}$

El propósito de este análisis es describir la seguridad de la intervención quirúrgica percutánea con tornillos en pacientes con fracturas de pelvis y acetábulo del Hospital de Traumatología «Dr. Victorio de la Fuente Narváez».

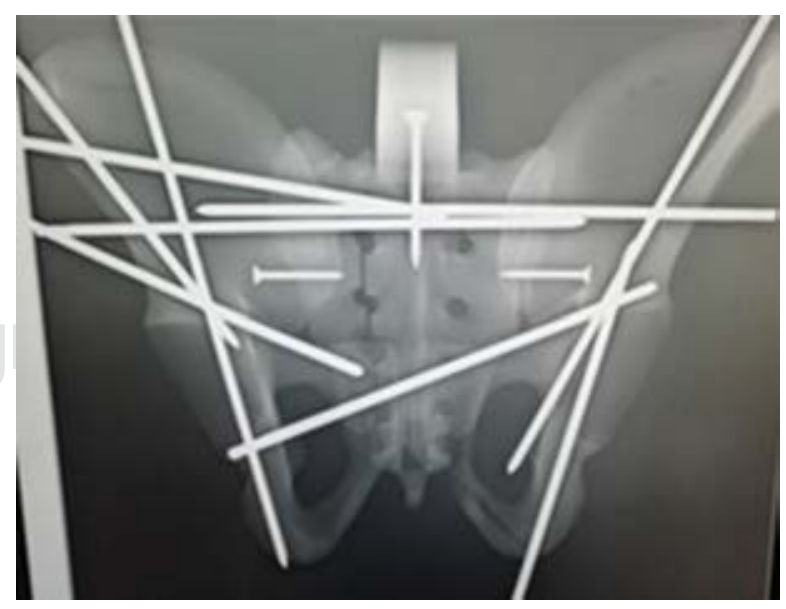

Figura 1: Configuración de la fijación percutánea en pelvis y acetábulo. 


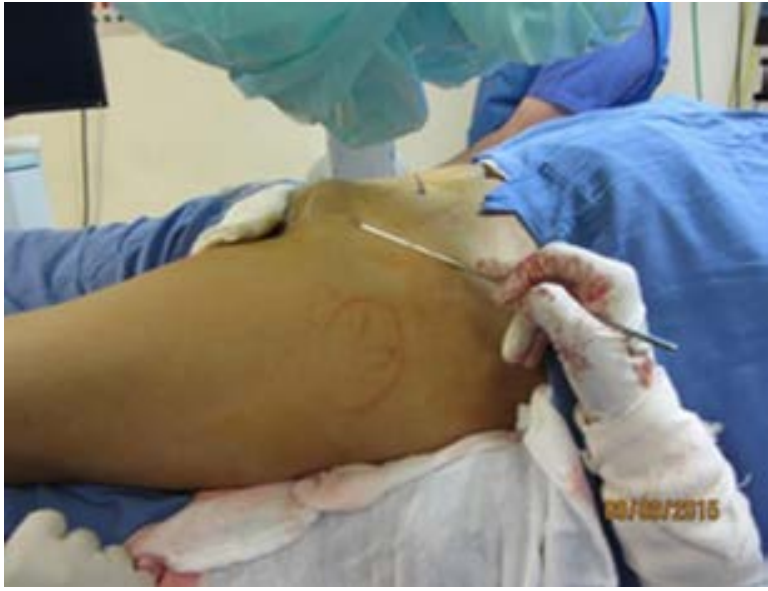

Figura 2: Preparación y colocación de tornillo percutáneo anterógrado en columna anterior de acetábulo.

\section{Material y métodos}

Se realizó un estudio observacional, retrospectivo, longitudinal y descriptivo. Se hizo revisión del registro de adultos hospitalizados con fracturas de pelvis y acetábulo del Servicio de Cadera, Pelvis y Acetábulo, con expediente electrónico o expediente físico completo del Hospital de Traumatología «Dr. Victorio de la Fuente Narváez» de enero del 2009 a diciembre del 2018, quienes cumplieron con los siguientes criterios de inclusión: pacientes con diagnóstico de fractura de pelvis y/o acetábulo no desplazadas, mínimamente desplazadas (hasta $3 \mathrm{~mm}$ ) que se reducen con maniobras de reducción cerrada, adultos con edad igual o mayor a 18 años, con expediente clínico completo a quienes se les dio seguimiento por consulta externa de hasta por un año. Se excluyeron aquellos pacientes menores de 18 años, con expediente clínico incompleto y que no tuvieron seguimiento por la consulta externa (Figura 5).

Se tomó como tamaño de la muestra la totalidad de los pacientes ingresados en el Servicio de Cadera y Pelvis del Hospital de Traumatología «Dr. Victorio de la Fuente Narváez» entre el periodo comprendido de enero 2009 a diciembre 2018 a los que se les practicó cirugía percutánea.

En el presente trabajo se evaluaron las variables sexo, edad, días de estancia en el hospital, muerte intrahospitalaria, tipo de fractura de pelvis y acetábulo con base en la nueva clasificación AO 2018, mecanismo de lesión el cual se dividió en un aspecto de baja energía, que se refiere a una caída de su plano de sustentación o menor de dos metros de altura, y otro de alta energía, en el cual se engloban: accidentes automovilísticos, accidentes en motocicleta, atropellamiento, caídas mayores a dos metros de altura y lesiones por aplastamiento. Otra variable a estudiar fueron los traumatismos asociados con fracturas de pelvis y acetábulo, entre las cuales se describen daños de cráneo o cuello, daños en tórax o en abdomen, lesiones en miembros torácicos, miembros pélvicos, traumatismos de columna y lesiones del aparato genitourinario. También se registró el porcentaje de complicaciones transoperatorias y postoperatorias. Por último, se valoró el grado de consolidación ósea con base en las radiografías valoradas en el seguimiento por la consulta externa de hasta al menos un año utilizando la clasificación de Montoya para determinar el grado de consolidación ósea.

\section{Resultados}

Se encontró información de 531 pacientes a los cuales se les realizó algún tipo de cirugía percutánea en fracturas de pelvis y/o acetábulo del Hospital de Traumatología «Dr. Victorio de la Fuente Narváez», del periodo comprendido de enero de 2009 a di-

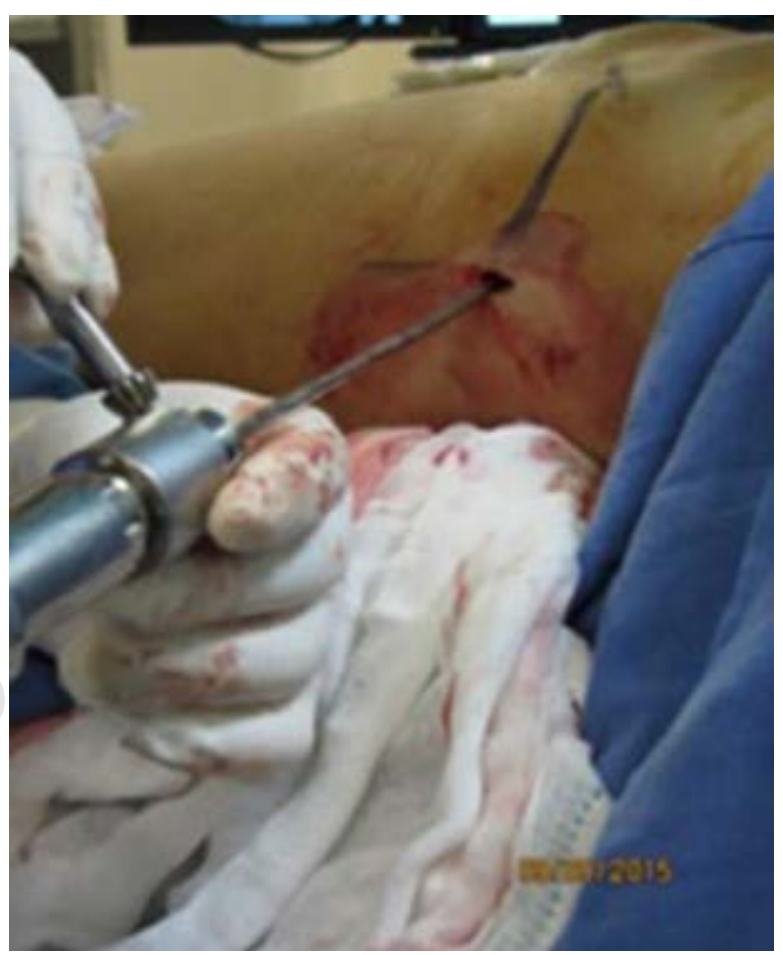

Figura 3: Colocación de tornillo iliosacro. 

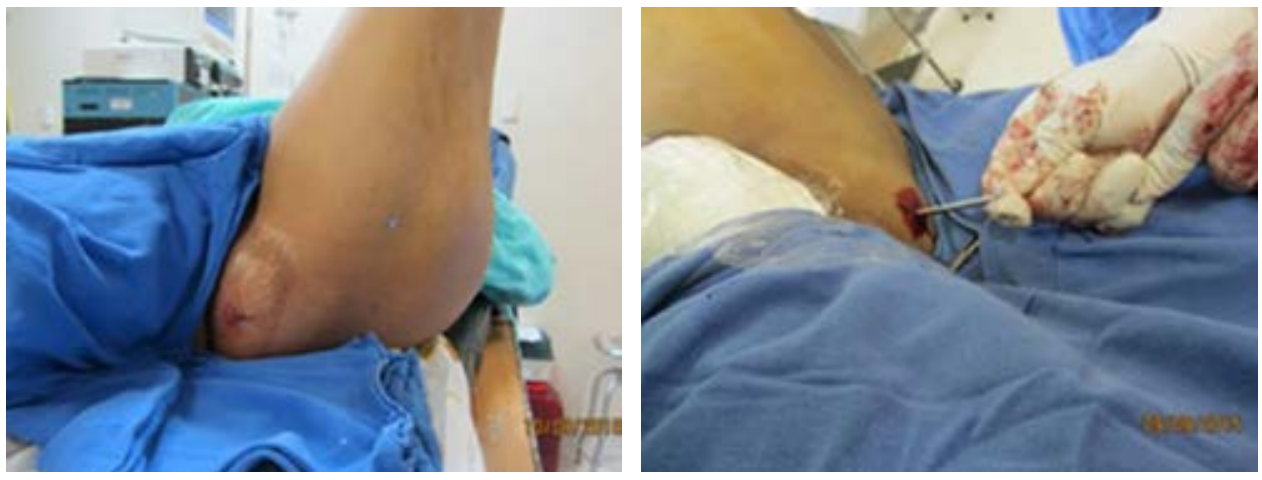

Figura 4:

Posición del paciente para colocación de tornillo percutáneo a columna posterior de acetábulo.

ciembre de 2018, de los cuales 454 cumplieron con los criterios de inclusión establecidos. De los 454 pacientes estudiados, el promedio de edad fue de 41.7 años, teniendo como mínimo de edad 18 años y como máximo de edad, un paciente con 92 años. Hubo 311 pacientes del sexo masculino (68.5\%) y 143 del sexo femenino (31.5\%) (Figura 6).

En relación con el tipo de fractura, se encontró que la más frecuente a la que se le practicó una operación percutánea fue a la fractura tipo AO 61B2 con un total de 173 pacientes (38.1\%), la segunda fractura más común fue la fractura de pelvis asociada con fractura de acetábulo con 61 pacientes (13.4\%), en tercer lugar está la fractura AO 61B1 con 49 pacientes (10.8\%), en cuarto sitio se encuentra la fractura $A O 61 \mathrm{C} 1 \mathrm{con}$ 37 afectados (8.1\%) y en quinto lugar la fractura $\mathrm{AO}$ $61 \mathrm{~B} 3$ con 36 pacientes $(7.9 \%)$. En relación con las fracturas de acetábulo puro a los que se les realizó este modelo de procedimiento las más usuales sometidas a tal método fueron las fracturas AO 62A3 con 21 afectados (4.6\%) y en el segundo puesto están las fracturas $\mathrm{AO} 62 \mathrm{~B} 1$ con 15 afectados (3.3\%).

El mecanismo de lesión de alta energía se presentó en 412 pacientes $(90.75 \%$ ) y en el de baja energía se acumularon 42 pacientes (9.25\%). En cuanto a los días de estadía intrahospitalaria, se obtuvo el registro mínimo de un día y el máximo de 70 días, siendo el promedio de 10.8 días de estancia en el hospital. De los 454 internados, a los que se les practicó cirugía percutánea, 15 pacientes (3.3\%) presentaron las siguientes complicaciones: malposición de material de osteosíntesis con ocho afectados (1.8\%), desanclaje o intolerancia de material de osteosíntesis en cinco pacientes $(1.1 \%)$ e infección del sitio quirúrgico en dos pacientes $(0.4 \%)$.

En relación con las lesiones asociadas con fracturas de pelvis y acetábulo, 218 pacientes (48\%) no tuvieron traumatismos relacionados con su fractura, mientras que 236 pacientes (52\%) tuvieron uno o más daños asociados en varios órganos o sistemas del cuerpo. De éstos se sumó un total de 393 lesiones asociadas, dentro de las cuales la más frecuente fue la lesión de miembros pélvicos con $89(23 \%)$ donde se incluyen fracturas o luxaciones de la misma extremidad, así como hematoma de Morel-Lavallée; en el segundo puesto aparecen las lesiones de los miembros torácicos con 72 (18\%), igualmente se incluyen fracturas o luxaciones de esta extremidad; en el tercer puesto de frecuencia se presentaron los daños en tórax con 61 (16\%) que abarcan trauma cerrado de tórax, hemotórax o neumotórax, fracturas costales y tórax inestable; en cuarto sitio están las lesiones en cráneo o cuello con 55 (14\%), donde hubo traumatismo craneoencefálico, trauma facial y ocular, en quinto lugar de frecuencia se observaron las lesiones en abdomen con 51 (13\%) como trauma cerrado de abdomen y cualquier lesión de órgano o víscera hueca intraabdominal, que requirió de algún procedimiento quirúrgico para su reparación; traumatismos en columna vertebral en sexto lugar con 39 (10\%), aquí se engloban el trauma raquimedular y la fractura y/o luxación de cualquier vértebra, y por último las lesiones del aparato genitourinario con $26(7 \%)$ en quienes se presentó lesión vesical o uretral principalmente.

La muerte intrahospitalaria sólo se presentó en dos pacientes $(0.44 \%)$ a los que se les realizó cirugía percutánea para fracturas de pelvis y/o acetábulo, el fallecimiento fue debido a que estos pacientes presentaron múltiples lesiones asociadas que fueron incompatibles con la vida, el resto 452 (99.56\%) de pacientes tuvieron su egreso hospitalario y seguimiento por consulta externa, de los cuales 100\% presentó un grado de consolidación ósea grados III y IV con base en la escala de Montoya. 


\section{Discusión}

La cirugía percutánea realizada en fracturas de pelvis y acetábulo en el Hospital de Traumatología «Dr. Victorio de la Fuente Narváez», de la UMAE Magdalena de las Salinas, ha aumentado en los últimos años debido a una mayor incidencia de este patrón de lesiones que requieren un manejo alternativo a la intervención abierta de pelvis y acetábulo. En el presente estudio, se encontró que la fractura más frecuente a la que se le practicó cirugía percutánea fue la fractura tipo AO 61B2 (38.1\%) (Figura 7), por otro lado, las fracturas por compresión lateral (AO 61B1, 61B2, 61B3) fue el mayor grupo al que se le efectuó tal procedimiento con $56.74 \%$. El segundo ejemplo de fractura más usual fue la fractura de pelvis asociada con fractura de acetábulo (13.4\%). En relación con las fracturas de acetábulo puro a los que se les practicó este tipo de método (Figura 8), se encontró que la fractura más habitualmente sometida a dicho procedimiento fueron las fracturas AO $62 \mathrm{A3}$ (4.6\%). Qoreishi y cols. ${ }^{9}$ en un artículo de cirugía percutánea, similar al aquí presentado, usan la clasificación de Young-Burgess y mencionan que el tipo de fractura más frecuente al que ellos realizaron la operación percutánea fue la fractura con mecanismo de compresión lateral con $28.7 \%$, seguido por fracturas aisladas de acetábulo con $21.7 \%$. De éstas, la más común fue la fractura transversa, posteriormente las fracturas de pelvis con compresión anteroposterior combinada con acetábulo con $20.3 \%$.

El mecanismo de lesión más reiterado en el presente trabajo fue el tipificado como de alta energía con $90.75 \%$, el resto fue por lesiones de baja energía con $9.25 \%$, donde se incluyeron daños por caídas de menos de 2 metros de altura. Boudissa y colaborado- res, ${ }^{10}$ en una investigación retrospectiva de 10 años de fracturas de pelvis y acetábulo, encontraron que el mecanismo de lesión más frecuente fue el de alta energía con $75 \%$, mientras que el tipo de baja energía se presentó en $25 \%$ de los casos, similar a este análisis, donde se observa que la mayor frecuencia de lesiones es por mecanismos de alta energía.

En relación con la estancia intrahospitalaria, se obtuvo un promedio de 10.8 días. Qoreishi y cols., en su texto sobre cirugía percutánea en fracturas de pelvis y acetábulo de 143 pacientes, tuvo una estadía en el hospital de $4 \pm 2$ días, un promedio menor al observado en el presente artículo. Ghosh y colegas ${ }^{11}$ registraron un promedio de 14.4 días en su investigación epidemiológica, donde incluyeron tratamiento conservador y cualquier tipo de intervención quirúrgica para fracturas de pelvis y acetábulo. En este estudio, la operación percutánea efectuada pudo combinarse con cirugía abierta en otro lugar diferente de la pelvis y/o acetábulo, por lo que el promedio de días de estancia intrahospitalaria aumentó y no se acerca a la cifra que maneja Qoreishi y colaboradores donde ellos utilizaron cirugía percutánea de pelvis y acetábulo pura. Además de que, en este trabajo, $52 \%$ de los pacientes presentó otras lesiones asociadas, que prolongaron la permanencia en el nosocomio debido a el tratamiento de éstas.

De los traumatismos asociados, los más frecuentes fueron los daños de miembro pélvico con $23 \%$, posteriormente, los daños de los miembros torácicos con $18 \%$, en tercer sitio, las lesiones en tórax con $16 \%$, en cuarto lugar de frecuencia se encontraron los traumatismos en cráneo o cuello con $14 \%$, lesiones en abdomen con $13 \%$, lesiones en columna $10 \%$, y por último los daños del aparato genitourinario con $7 \%$. Gosh y cols. reportan traumatismos asociados de miembros pélvicos en $29 \%$, lesiones en abdomen

Figura 5:

Paciente con fractura de ambas columnas de acetábulo a quien se le realizó fijación percutánea.
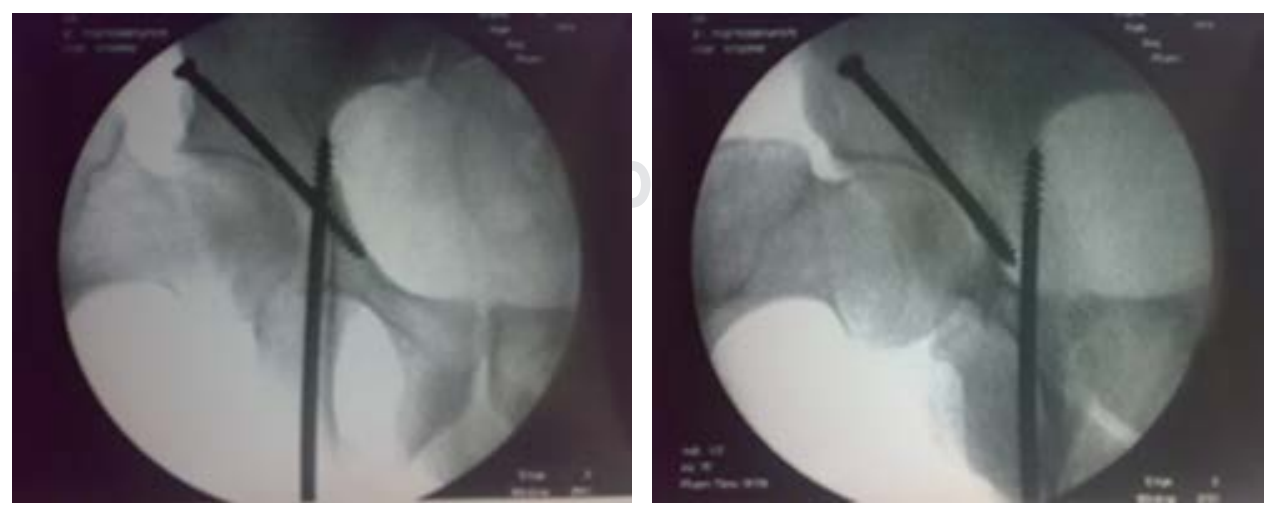
Tornillos percutáneos colocados anualmente

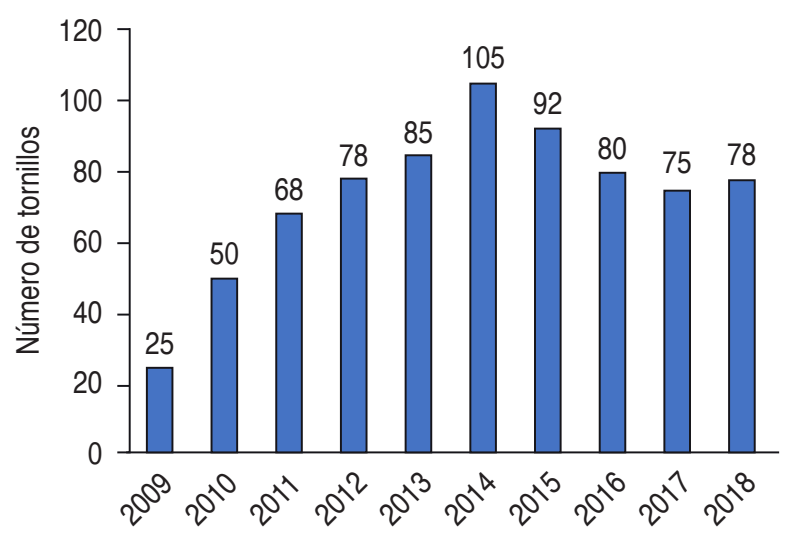

Figura 6: Número de tornillos percutáneos colocados anualmente en el Hospital de Traumatología «Dr. Victorio de la Fuente Narváez», en el Servicio de Cadera y Pelvis, 2009-2018.

$28 \%$, lesiones en cráneo $22.7 \%$, daños urogenitales $22.7 \%$, y lesiones en tórax $10.7 \%$, de estas lesiones reportadas por Gosh y colegas, en el presente artículo se tiene un porcentaje parecido en la lesión asociada más habitual que es el traumatismo de los miembros pélvicos, las demás lesiones asociadas pueden variar debido a que la muestra en su análisis es menor en comparación a la presentada aquí, además de que, en su artículo, Gosh y colaboradores incluyeron cirugía abierta, lo que puede modificar el tipo de fractura y relacionarse con otra forma de lesión asociada. Yang y su equipo ${ }^{12}$ en un reporte epidemiológico de 10 años en Taiwán, encontraron que la lesión más usual asociada con fracturas de pelvis y acetábulo son las lesiones de los miembros pélvicos con $21.5 \%$, en segundo lugar encontraron las lesiones del tórax y columna con $20.9 \%$, seguido de las lesiones en miembros superiores con $18 \%$, daños en cráneo con $17 \%$ y las lesiones en abdomen con $11 \%$.

En el presente estudio, sólo 15 pacientes (3.3\%) presentaron alguna complicación transquirúrgica y postquirúrgica, entre las que se encontró: malposición de material de osteosíntesis en ocho pacientes $(1.8 \%)$, desanclaje o intolerancia de material de osteosíntesis en cinco pacientes (1.1\%) e infección del sitio quirúrgico en dos pacientes (0.4\%); Qoreishi y su equipo sólo reportaron un paciente con malposición del material de osteosíntesis y un paciente con infección del sitio quirúrgico, en total $1.4 \%$ de complicaciones de sus 143 pacientes, y no reportan lesiones neurológicas. Son porcentajes similares a los descritos en el presente trabajo. Eckardt y colaboradores ${ }^{13}$ tuvieron un índice de dificultades transoperatorias del $4 \%$ (un paciente con lesión vascular y otro con lesión neurológica), en su análisis de 50 pacientes, además, en el seguimiento de éstos, reportan que tuvieron $18 \%$ de los pacientes con aflojamiento de material de osteosíntesis que requirieron de reintervenciones quirúrgicas. Osterhoff y su equipo, ${ }^{14}$ en su estudio de 38 pacientes a los que se les realizó fijación percutánea a lesiones del anillo pélvico posterior, reportan malposición del tornillo en $10.5 \%$, lesión nerviosa en $5.3 \%$ y no unión en $2.6 \%$, cifras muy por encima de lo reportado en este artículo. Zwingmann y cols. ${ }^{15}$ en un metaanálisis determinan que el porcentaje de malposición con técnica convencional de fluoroscopia es de $2.6 \%$, cifra similar a la de $1.8 \%$ obtenida por los autores de este texto.

En relación con la mortalidad en las lesiones del anillo pélvico, la literatura presenta índices con amplia variación, y los autores siempre enfatizan la asociación de estas tasas con los traumatismos asociados y la gravedad de la lesión del anillo. En el presente trabajo, se tuvo un porcentaje de mortalidad de $0.44 \%$, sólo dos pacientes fallecieron durante su estancia intrahospitalaria, ellos fallecieron por presentar múltiples daños asociados y datos de choque séptico. Yoshihara, ${ }^{16}$ en un reporte epidemiológico sobre tal tipo de lesión, informó una tasa de mortalidad hospitalaria en pacientes con fractura pélvica inestable de $8.3 \%$. Pereira y

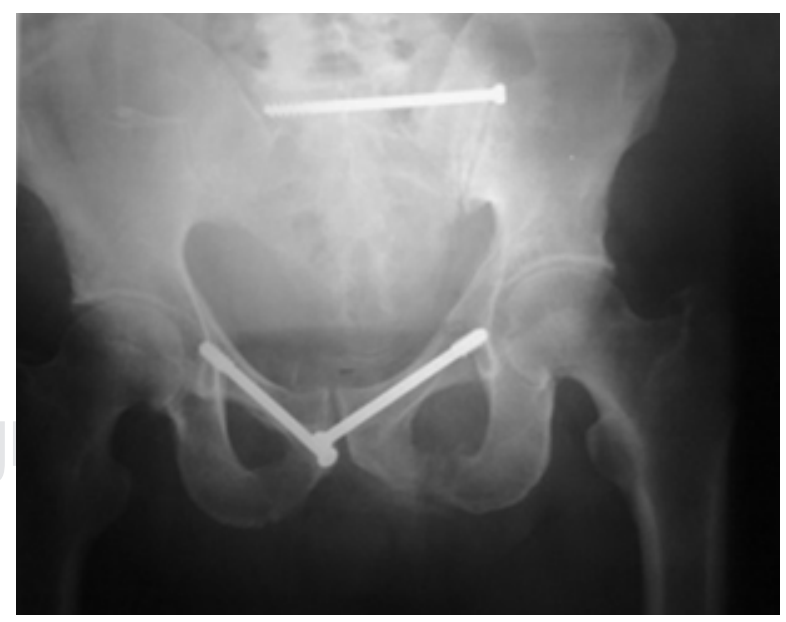

Figura 7: Paciente con fractura de pelvis A0 61B2.1*b, postoperado de fijación percutánea con tornillo iliosacro y tornillo retrogrado a ambas ramas iliopúbicas. 


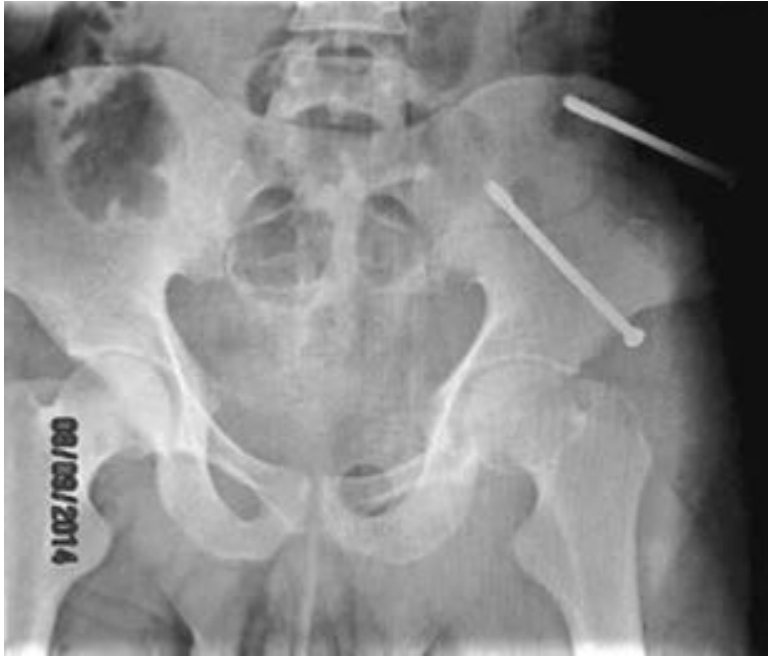

Figura 8: Paciente con fractura de iliaco, postoperado de fijación percutánea.

colegas $^{17}$ reportan una tasa de mortalidad de $3 \%$ asociada con mecanismos de alta energía. Se considera que el factor más importante en la baja tasa de mortalidad observada en este estudio fue el alto número de casos de fracturas $\mathrm{AO} 61 \mathrm{~B} 1, \mathrm{AO}$ 61B2, AO 61B3, que generalmente no tienen tantas lesiones o complicaciones asociadas.

En este reporte, $100 \%$ de los pacientes tuvo una consolidación ósea de grados III y IV en la escala de Montoya en el seguimiento a un año por la consulta externa del servicio de cadera, pelvis y acetábulo. Qoreishi y cols. menciona que $100 \%$ de sus pacientes presentó consolidación ósea a los tres meses de seguimiento, y podían realizar apoyo total de las extremidades en su última consulta de seguimiento, cifra igual a la mostrada por el presente estudio.

\section{Conclusiones}

La cirugía percutánea en fracturas de pelvis y acetábulo es un procedimiento quirúrgico seguro para pacientes con fracturas no desplazadas o mínimamente desplazadas, ya que se asocia con una menor morbilidad, tiene un alto porcentaje de consolidación ósea, puede usarse como complemento a una reducción abierta y fijación interna en otro segmento de la pelvis y acetábulo, o como tratamiento definitivo en pacientes con múltiples comorbilidades, a fin de dar estabilidad a el anillo pélvico. Es un recurso que requiere de entrenamientos especiales, una curva de aprendizaje prolongada y una planificación preope- ratoria adecuada. Además, hay que considerar que tiene una alta tasa de exposición a radiación por parte del cirujano.

\section{Referencias}

1. Prieto E, Giráldez M. Efectividad y seguridad del tratamiento percutáneo de las fracturas del anillo pélvico. España: Tecnologías Sanitarias de Andalucía; 2011.

2. Giannoudis P, Tzioupis C. Percutaneous fixation of the pelvic ring: an update. J Bone Joint Surg. 2007; 89 (2): 145-154.

3. Von Keudell A, Tobert D, Rodriguez E. Percutaneous fixation in pelvic and acetabular fractures: understanding evolving indications and contraindications. Oper Tech Orthop. 2015; 25: 248-255.

4. Caviglia H, Mejail A, Landro ME, Vatani N. Percutaneous fixation of acetabular fractures. EFORT Open Rev. 2018; 3 (5): 326-334.

5. Bozzio AE, Wydra FB, Mitchell JJ, Ackerson RM, Mauffrey C. Percutaneous fixation of anterior and posterior column acetabular fractures. Orthopedics. 2014; 37 (10): 675-678.

6. Routt ML Jr, Nork SE, Mills WJ. Percutaneous fixation of pelvic ring disruptions. Clin Orthop Relat Res. 2000; (375): 15-29.

7. Graves ML, Routt ML Jr. Iliosacral screw placement: are uniplanar changes realistic based on standard fluoroscopic imaging? J Trauma. 2011; 71 (1): 204-208; discussion 208.

8. Morshed S, Choo K, Kandemir U, Kaiser SP. Internal fixation of posterior pelvic ring injuries using iliosacral screws in the dysmorphic upper sacrum. JBJS Essent Surg Tech. 2015; 5 (1): e3.

9. Qoreishi M, Seyyed Hosseinzadeh HR, Safdari F. Clinical results of percutaneous fixation of pelvic and acetabular fractures: a minimally invasive internal fixation technique. Arch Bone Jt Surg. 2019; 7 (3): 284-290.

10. Boudissa M, Francony F, Kerschbaumer G, Ruatti S, Milaire $\mathrm{M}$, Merloz $\mathrm{P}$, et al. Epidemiology and treatment of acetabular fractures in a level-1 trauma centre: Retrospective study of 414 patients over 10 years. Orthop Traumatol Surg Res. 2017; 103 (3): 335-339.

11. Ghosh S, Aggarwal S, Kumar V, Patel S, Kumar P. Epidemiology of pelvic fractures in adults: Our experience at a tertiary hospital. Chin J Traumatol. 2019; 22 (3): 138-141.

12. Yang NP, Chan CL, Chu D, Lin YZ, Lin KB, Yu CS, et al. Epidemiology of hospitalized traumatic pelvic fractures and their combined injuries in Taiwan: 2000-2011 National Health Insurance data surveillance. Biomed Res Int. 2014; 2014: 878601.

13. Eckardt H, Egger A, Hasler RM, Zech CJ, Vach W, Suhm $\mathrm{N}$, et al. Good functional outcome in patients suffering fragility fractures of the pelvis treated with percutaneous screw stabilisation: Assessment of complications and factors influencing failure. Injury. 2017; 48 (12): 2717-2723.

14. Osterhoff G, Ossendorf C, Wanner GA, Simmen HP, Werner $\mathrm{CM}$. Percutaneous iliosacral screw fixation in S1 and S2 for posterior pelvic ring injuries: technique and perioperative complications. Arch Orthop Trauma Surg. 2011; 131 (6): 809813.

15. Zwingmann J, Hauschild O, Bode G, Sudkamp NP, Schmal H. Malposition and revision rates of different imaging modalities for percutaneous iliosacral screw fixation following pelvic fractures: 
a systematic review and meta-analysis. Arch Orthop Trauma Surg. 2013; 133 (9): 1257-1265.

16. Yoshihara H, Yoneoka D. Demographic epidemiology of unstable pelvic fracture in the United States from 2000 to 2009: trends and in-hospital mortality. J Trauma Acute Care Surg. 2014; 76 (2): 380-385.

17. Pereira GJC, Damasceno ER, Dinhane DI, Bueno FM, Leite $\mathrm{JBR}$, Ancheschi BDC. Epidemiology of pelvic ring fractures and injuries. Rev Bras Ortop. 2017; 52 (3): 260-269.

\section{Conflicto de intereses}

Los autores han tenido en cuenta las instrucciones y las responsabilidades éticas, cumplen con los requisitos de autoría y declaran la no existencia de conflicto de intereses en aquellas actividades relacionadas con el artículo. 